\title{
Osteochondritis Dissecans Lesions in Family Members: Does a Positive Family History Impact Phenotypic Potency?
}

\author{
Alex L. Gornitzky MD, R. Justin Mistovich MD, Brittany Atuahuene BA, \\ Eileen P. Storey BA, Theodore J. Ganley MD
}

Published online: 6 September 2016

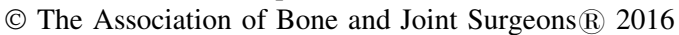

\begin{abstract}
Background Although repetitive microtrauma and athletic overuse patterns are most commonly associated with osteochondritis dissecans (OCD), recent studies have identified a potential genetic predisposition for OCD. Several case series have documented family pedigrees that support autosomal-dominant inheritance, but the families in these studies were all selected as a result of unique histories that may not accurately represent OCD inheritance patterns at large. Because there has been little investigation beyond these case reports, we aimed to
\end{abstract}

Each author certifies that he or she, or a member of his or her immediate family, has no funding or commercial associations (eg, consultancies, stock ownership, equity interest, patent/licensing arrangements, etc) that might pose a conflict of interest in connection with the submitted article.

All ICMJE Conflict of Interest Forms for authors and Clinical Orthopaedics and Related Research $\AA$ editors and board members are on file with the publication and can be viewed on request.

Each author certifies that his or her institution approved or waived approval for the human protocol for this investigation and that all investigations were conducted in conformity with ethical principles of research.

This work was performed at the Children's Hospital of Philadelphia, Philadelphia, PA, USA.

A. L. Gornitzky, B. Atuahuene, E. P. Storey, T. J. Ganley ( $\square)$

Division of Orthopaedics, The Children's Hospital of

Philadelphia, Philadelphia, PA, USA

e-mail: ganley@email.chop.edu

\section{A. L. Gornitzky}

Perelman School of Medicine, University of Pennsylvania, Philadelphia, PA, USA

\section{R. J. Mistovich}

Department of Orthopaedic Surgery, Case Western Reserve University School of Medicine, Cleveland, OH, USA describe a broader, more representative pattern of OCD inheritance applicable to all affected patients.

Questions/Purposes (1) What proportion of patients treated for OCD of the knee have one or more immediate and/or extended family members with a history of OCD lesions? (2) Do patients with more phenotypically potent lesions, which we defined as patients with bilateral OCD lesions or patients who have undergone multiple procedures for OCD, have a higher frequency of affected relatives than those with less potent lesions?

Methods This retrospective study queried patient databases, diagnosis codes (International Classification of Diseases, 9th Revision), and surgical logs at a regional, tertiary care children's hospital to identify all patients treated over a 10-year period (March 2004-March 2014) by the senior author for OCD of the knee. All patients aged 0-18 years at the time of diagnosis were included. At our institution, patients with intact lesions are treated with a trial of conservative therapy; conversely, patients with a break in the articular cartilage and/or loose fragments of bone/cartilage are treated surgically. There were no OCDspecific contraindications to surgery. This search identified 543 patients. After patient identification, a questionnaire was designed that asked for the number, age, and gender of all immediate family members and the history of OCD lesions in any family member (immediate or extended). For all positive family members, patients were further queried regarding relevant clinical details to affirm a history of OCD. Patients were contacted by mailed questionnaires and phone calls for survey completion. All 543 patients received the survey, of which $103(19 \%)$ responded to it and were included here. Responders were approximately 1 year younger than nonresponders; there was no difference in gender distribution. A retrospective chart review was then conducted 
to collect demographic information, phenotypic disease severity, and treatment details. Of the 103 included patients, 20 patients (19\%) with unilateral lesions were managed nonoperatively ("conservative" group), 50 patients (49\%) had unilateral lesions advanced to surgery ("unilateral" group), 21 patients $(20 \%)$ had bilateral lesions managed either conservatively or surgically ("bilateral" group), and 12 patients (12\%) underwent multiple procedures for the same lesion ("multiple" group). Of those included, 75 patients $(73 \%)$ were treated surgically. With the numbers available, there were no baseline differences among the groups in terms of gender, lesion laterality, lesion location, or number of secondary procedures at the time of the initial surgical intervention. Results In total, 14 of 103 (14\%) patients treated for OCD in this study had an immediate and/or extended family member with a history of OCD lesions. This included four of $20(20 \%)$ patients in the conservative group, five of 50 $(10 \%)$ in the unilateral group, four of $21(19 \%)$ in the bilateral group, and one of $12(8 \%)$ in the multiple group. With the numbers available, we did not identify a higher proportion of immediate and/or extended family members with a positive history of OCD in those patients with phenotypically potent lesions (bilateral and multiple) as compared with those with patients phenotypically less potent lesions (conservative and unilateral; five of 33 [15\%] versus nine of 70 [13\%]; odds ratio, 1.2 ; 95\% confidence interval, 0.4-3.9; $\mathrm{p}=0.751$ ).

Conclusions In this broad, heterogeneous cohort of pediatric patients with $\mathrm{OCD}$, the proportion of patients with a positive family history of OCD was $14 \%$, which appeared to be much higher than the reported prevalence of OCD in the general population according to prior research. Therefore, our data provide preliminary support for a familial inheritance pattern for OCD, suggesting that further clinical, biologic, and genomic investigation might help to improve our understanding of who is at highest risk for OCD and what moderating factors might influence their disease severity and risk of progression. Furthermore, our data suggest that expanded patient education and screening regarding inheritance patterns might enhance identification of potential familial disease burden and improve access to timely and appropriate treatment.

Level of Evidence Level IV, prognostic study.

\section{Introduction}

Osteochondritis dissecans (OCD) is a relatively rare cause of knee pain with an estimated incidence in the general population between $0.01 \%$ and $4 \%$ [3, 10, 13, 16]. Despite increased awareness of the progression of OCD, the underlying etiology of this condition remains unknown. In the earliest description of OCD in 1888, Konig proposed an inflammatory etiology; however, this has since been refuted and replaced by several other theories [11]. These include repetitive microtrauma and secondary effects associated with vascular insufficiency, avascular necrosis, and genetic predisposition [6, 14, 23]. Although repetitive microtrauma has become the most accepted cause of OCD because of the rising incidence of this disorder among athletes, several recent reports have also investigated an underlying genetic contribution [4].

Although Petrie first concluded that OCD does not have a familial association in 1977, multiple case reports have been published since then that demonstrate a potentially heritable genetic component to the etiology of OCD [20]. Specifically, many of these recent case reports have described OCD lesions in sets of monozygotic twins, including some in which the appearance of the lesions themselves is identical $[5,9,15,17,19,21]$. In further support of a genetic etiology, OCD lesions have been cited as a component of several syndromic conditions. For example, researchers have identified a specific mutation in the type IX collagen gene that results in a form of multiple epiphyseal dysplasia with associated OCD lesions [8]. Al Kaissi et al. [1] described a family with a subset of Stickler syndrome in which OCD lesions were a constant syndromic component. This was linked to mutations in type II and XI collagen and inherited in an autosomal-dominant pattern. In a less severe case, researchers found a fivegeneration family in Sweden with disproportionate short stature, early osteoarthritis, and multiple OCD lesions inherited in an autosomal-dominant pattern [24]. Several other authors have described familial cases of OCD lesions associated with short stature and multiple lesion sites $[12,18,25]$.

Despite the suggested genetic link in studies of isolated OCD lesions in monozygotic twins and in syndromic occurrences, more research is needed to determine if a less severe genetic mutation or set of mutations exists that results in heritable OCD lesions without a syndromic association. To further this investigation, we sought to assess the incidence of OCD among family members of treated patients and further subcategorize inheritance based on the type and severity of lesion.

Therefore, we asked: (1) What proportion of patients treated for OCD of the knee have one or more immediate and/or extended family members with a history of OCD lesions? (2) Do patients with more phenotypically potent lesions, which we defined as patients with bilateral OCD lesions or patients who have undergone multiple procedures for OCD, have a higher frequency of affected relatives than those with less severe lesions? 


\section{Materials and Methods}

Institutional review board approval was obtained before starting this retrospective prognostic study. The Children's Hospital of Philadelphia patient databases, diagnosis codes (International Classification of Diseases, 9th Revision), and surgical $\operatorname{logs}$ were queried to identify all patients treated over a 10-year period (March 2004-March 2014) by the senior author (TJG) for OCD of the knee. To facilitate uniform comparison, only OCD lesions of the knee were considered, although patient reports of family members with lesions in other locations were included. All patients aged $0-18$ years at the time of initial diagnosis were included. At our institution, all patients with intact lesions are first treated with a trial of conservative therapy (6 weeks of weightbearing as tolerated with a brace locked in extension). Subsequently, bracing is discontinued and patients are permitted to walk but not run or jump (ie, activity restriction) for 6 weeks. Patients then follow up with repeat radiographs at 3-month intervals. Conversely, patients with a break in the articular cartilage and/or loose fragments of bone/cartilage are treated surgically without a trial of conservative therapy. There were no OCD-specific contraindications to surgery. This search identified 543 patients. After patient identification, a questionnaire was designed that asked for the number, age, and gender of all immediate family members and the history of OCD lesions in any family member (immediate or extended). Patients were also queried regarding athletic activity, including sport played and hours of participation per week. For all positive family members, patients were further queried regarding relevant clinical and surgical details to affirm a positive history of OCD. Patients were contacted by mailed questionnaires and phone calls for survey completion. Because this was considered a preliminary study intended to guide future multicenter clinical and/or genomic studies, a goal of approximately 100 enrolled patients was targeted. Given limited previous work on this subject, an a priori power analysis was not completed. Therefore, all 543 patients received the survey, and patient enrollment was stopped at $103(19 \%)$ patients. A retrospective chart review was then conducted to collect demographic information, phenotypic disease severity, and treatment details. Of the 103 included patients, 20 patients (19\%) with unilateral lesions were managed nonoperatively ("conservative" group), 50 patients (49\%) had unilateral lesions advanced to surgery ("unilateral" group), 21 patients (20\%) had bilateral lesions managed either conservatively or surgically ("bilateral" group), and 12 patients (12\%) underwent multiple procedures for the same lesion ("multiple" group). Overall, 28 of 103 (27\%) included patients were managed nonoperatively (20 unilateral, eight bilateral) and $75(73 \%)$ were managed operatively. Operatively managed patients were 2 years older than patients managed conservatively (14 years versus 12 years, mean difference [MD] 1.6 years, $95 \%$ confidence interval $[\mathrm{CI}] 0.7-2.5$, p value $<$ 0.001). This cohort included two sets of siblings (each counted individually): one set of concordant monozygotic twins (unilateral and bilateral) and one brother and sister (conservative and bilateral, respectively). Therefore, our cohort included 103 patients representing 101 families. Patients were further subdivided into phenotypically potent lesions (bilateral and multiple) and phenotypically nonpotent lesions (conservative and unilateral). With the numbers available, there were no baseline differences among the two potency groups in terms of gender, lesion laterality, lesion location (medial femoral condyle versus all other locations), number of secondary procedures at the time of the initial surgical intervention, or number of immediate family members (Table 1). Although of unclear clinical significance, patients with phenotypically potent lesions were 1 year younger than those with phenotypically nonpotent lesions (mean 13 years versus 14 years; MD -1 years, $95 \% \mathrm{CI}-0.2$ to -0.2 , $\mathrm{p}$ value $=0.020$ ). The survey did not query patients regarding race and ethnicity, and patient responses to athletic activity were too varied and/or inconsistent to make meaningful, cohort-wide conclusions.

All 543 patients received the survey, of which 103 (19\%) responded to it and were included here. Responders were 1 year younger than nonresponders (13 years versus 14 years, MD -0.8 years, $95 \% \mathrm{CI}-1.3$ to -0.2 , $\mathrm{p}$ value $0.005)$. There was no difference in gender distribution (77 of 103 [75\%] male responders versus 311 of 440 [71\%] male nonresponders, odds ratio [OR] $1.2,95 \%$ CI $0.8-2.0$, $\mathrm{p}$ value $=0.405)$. Because a retrospective chart was only completed on included patients, differences in treatment type were unable to be assessed. All phone calls were made by the first author (ALG) in a blinded fashion before any retrospective chart review to minimize administrator bias. Positive family history was affirmed positive in patients able to provide an unprompted clinical course and treatment history consistent with OCD. To identify as many falsely positive histories as possible (eg, "my cousin had some type of knee injury that required surgery"), all questionnaires (phone and mail) included further queries regarding available clinical and/or surgical treatment details; unclear or vague histories were not included. Given the preliminary nature of this study, medical records and/or imaging were not reviewed for positive family members, and no family members were contacted and/or brought into the clinic for additional imaging and/or evaluation.

Comparative analysis was performed using using Fisher's exact/chi-square tests or Student's t-test, as indicated. Categorical variables are reported as proportions (numerator/denominator and percentage) with their associated effect size (OR) and 95\% CI; continuous variables are 


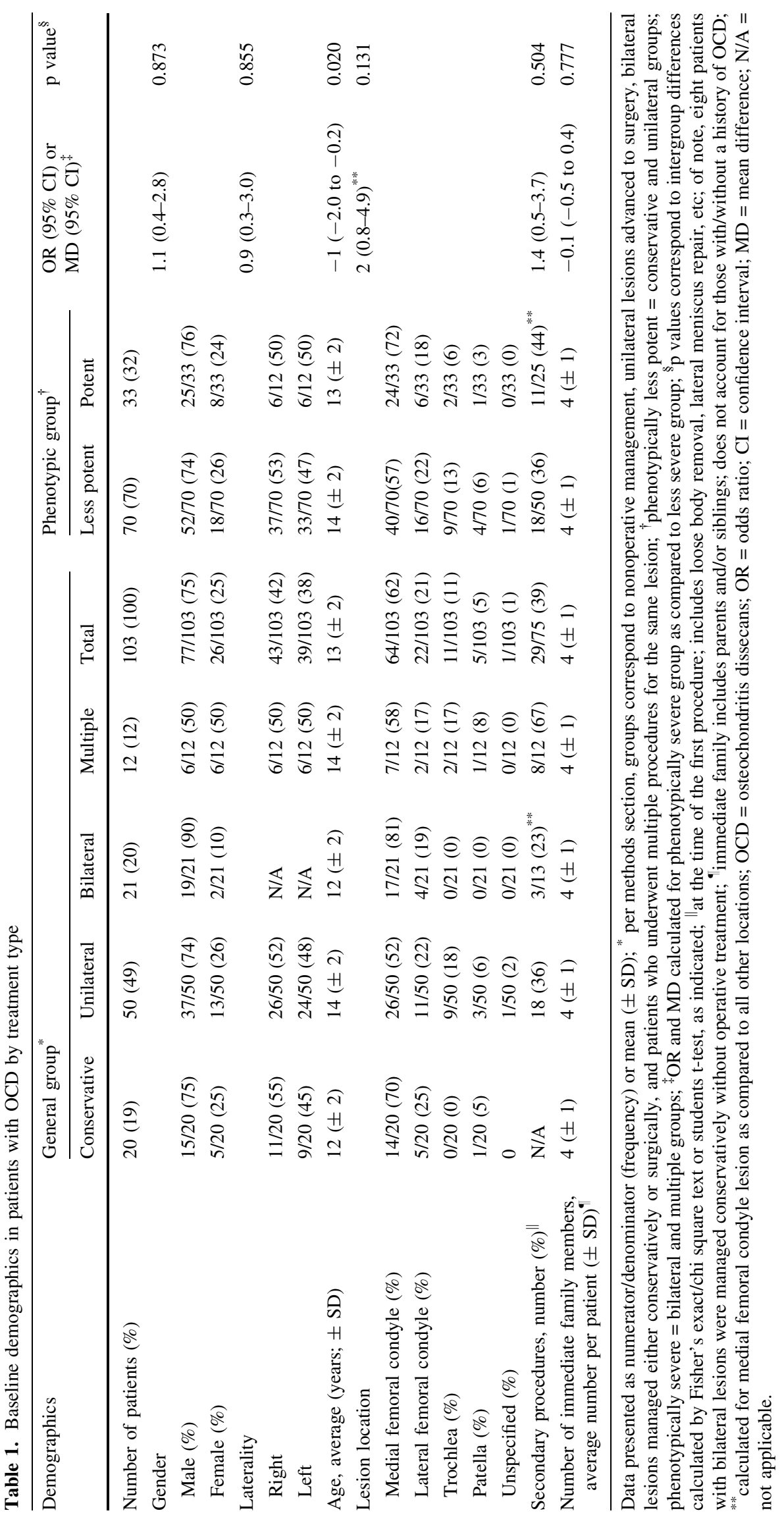


presented with a measure of central tendency (mean), spread (SD), and difference (MD and 95\% CI). An $\alpha$ level of 0.05 was used for all tests. Given the limited number of patients included in this preliminary study and the small intergroup differences, we had insufficient statistical power to resolve between-group differences. Given an alpha of $5 \%$, a power of $80 \%, 103$ included patients, and our detected proportion of 0.13 in the phenotypically less severe group, we would have needed to detect a difference (delta) in proportion of 0.25 between study groups to conclude that phenotypically severe disease was statistically associated with an increased proportion of patients with a positive family history.

\section{Results}

In total, 14 of 103 (14\%) patients treated for OCD in this study had an immediate and/or extended family member with a history of OCD lesions (Table 2). This included four of $20(20 \%)$ patients in the conservative group, five of 50 $(10 \%)$ in the unilateral group, four of $21(19 \%)$ in the bilateral group, and one of $12(8 \%)$ in the multiple group (Table 1). Only one patient had a family history notable for more than two successive generations positive for OCD, and no patients noted more than two other combined immediate and extended family members with a history of OCD.

With the numbers available, we did not identify a higher proportion of immediate and/or extended family members with a positive history of OCD in those patients with phenotypically potent lesions (bilateral and multiple) as compared with those patients with phenotypically less potent lesions (conservative and unilateral; five of 33 [15\%] versus nine of 70 [13\%], OR 1.2, 95\% CI, 0.4-3.9, p $=0.751)$. Additionally, we did not identify a difference in either the mean number of immediate family members between these two groups (four members $[ \pm 1]$ versus four members $[ \pm 1]$; $\mathrm{MD}-0.1,-0.5$ to $0.4, \mathrm{p}=0.777$ ) or the proportion of immediate family members with a positive history of OCD (two of 33 [6\%] versus six of 70 [9\%], OR $0.7,0.1-3.6, \mathrm{p}=0.658)$.

\section{Discussion}

Although repetitive microtrauma and athletic overuse patterns are most commonly associated with OCD, recent studies have identified a potential genetic predisposition for OCD. Several case series have documented family pedigrees that support autosomal-dominant inheritance, but the families in these studies were all selected as a result of unique histories that may not accurately represent OCD inheritance patterns at large. Because there has been little investigation beyond these case reports, we aimed to describe a broader, more representative pattern of OCD inheritance applicable to all affected patients. Additionally, we sought to evaluate whether a positive family history for OCD is associated with increased phenotypic disease

Table 2. Patients with a positive family history for OCD

\begin{tabular}{|c|c|c|c|c|c|c|c|c|c|}
\hline \multirow[t]{2}{*}{ Demographics } & \multicolumn{5}{|c|}{ General group ${ }^{*}$} & \multicolumn{2}{|c|}{ Phenotypic group $^{\dagger}$} & \multirow{2}{*}{$\begin{array}{l}\text { OR }(95 \% \mathrm{CI}) \text { or } \\
\mathrm{MD}(95 \% \mathrm{CI})^{*}\end{array}$} & \multirow[t]{2}{*}{$\mathrm{p}$ value } \\
\hline & Conservative & Unilateral & Bilateral & Multiple & Total & Less potent & Potent & & \\
\hline Number of patients (\%) & $20(19)$ & $50(49)$ & $21(20)$ & $12(12)$ & $103(100)$ & $70(70)$ & $33(32)$ & & \\
\hline $\begin{array}{l}\text { Number of immediate family } \\
\text { members, average number per } \\
\text { patient }( \pm \text { SD })\end{array}$ & $4( \pm 1)$ & $4( \pm 1)$ & $4( \pm 1)$ & $4( \pm 1)$ & $4( \pm 1)$ & $4( \pm 1)$ & $4( \pm 1)$ & $-0.1(-0.5$ to 0.4$)$ & 0.777 \\
\hline $\begin{array}{l}\text { Positive immediate family history, } \\
\text { number }(\%)\end{array}$ & $4 / 20(20)$ & $2 / 50(4)^{\bullet}$ & $2 / 21(10)$ & $0 / 12(0)$ & 8/103 (8) & $6 / 70(9)^{\bullet}$ & $2 / 33(6)$ & $0.7(0.1-3.6)$ & 0.658 \\
\hline $\begin{array}{l}\text { Positive extended family history, } \\
\text { number }(\%)\end{array}$ & 0/20 (0) & $4 / 50(8)^{\uparrow}$ & $2 / 21(10)$ & $1 / 12(8)$ & $7 / 103(7)$ & $4 / 70(6)^{\bullet}$ & $3 / 33(9)$ & $1.7(0.3-7.8)$ & 0.529 \\
\hline $\begin{array}{l}\text { Positive immediate and/or extended } \\
\text { family history, number }(\%)\end{array}$ & $4 / 20(20)$ & $5 / 50(10)^{\bullet}$ & $4 / 21(19)$ & $1 / 12(8)$ & 14/103 (14) & $9 / 70(13)^{\top}$ & $5 / 33(15)$ & $1.2(0.4-3.9)$ & 0.751 \\
\hline
\end{tabular}

Data presented as numerator/denominator (frequency) or mean $( \pm \mathrm{SD}) ;{ }^{*}$ per methods section, groups correspond to nonoperative management, unilateral lesions advanced to surgery, bilateral lesions managed either conservatively or surgically, and patients who underwent multiple procedures for the same lesion; ${ }^{\dagger}$ phenotypically nonpotent $=$ conservative + unilateral groups; phenotypically potent $=$ bilateral + multiple groups; ${ }^{\ddagger} \mathrm{OR}$ and MD calculated for phenotypically severe group as compared with nonsevere group;. ${ }^{\S} \underline{p}$ values correspond to intergroup differences calculated by Fisher's exact/chi square text or Student's t-test, as indicated; “immediate family includes parents and/or siblings; does not account for those with/without a history of OCD; one patient in the unilateral group had positive immediate family history and a positive extended family history. Therefore, this patient was counted twice in each of these individual groups, but only once in the combined group; OCD = osteochondritis dissecans; $\mathrm{OR}=$ odds ratio $\mathrm{CI}=$ confidence interval; $\mathrm{MD}=$ mean difference. 
potency. In this study, we found that $14 \%$ of patients with OCD have a family history of the condition. This seems higher than the incidence reported in the general population, which ranges from $0.01 \%$ to $4 \%[3,10,13,16]$. Contrary to anecdotal reports, however, our results did not demonstrate that patients with increased phenotypic disease severity are more likely to have a positive family history for disease.

The validity of a survey study depends in large measure on two things: the degree to which the population surveyed represents the larger population of interest and the degree to which those who responded represent the larger population of interest. Regarding the former, our data were collected from a single, large urban institution in the Northeastern United States. Therefore, our patient population may not accurately reflect other centers in terms of racial and ethnic diversity or baseline athletic activity, each of which may affect the documented proportion of relatives with OCD. Nevertheless, as an academic tertiary referral center, our hospital serves as a very large catchment area for pediatric patients diagnosed with OCD, thus increasing the potential demographic diversity of our patient population. Although we attempted to collect athletic activity and participation from included patients, survey responses to this specific question were too varied and/or incomplete to make meaningful, cohort-wide conclusions (eg, only writing "soccer" in response to the query regarding sports activity, level of play, and hour per week/weeks per year). This study's questionnaires also did not include information on race, ethnicity, or socioeconomic status, each of which could have affected disease severity and access to care. Overall, however, our patient cohort had clinical parameters comparable to those observed by Kessler et al. in their population-based study of pediatric OCD of the knee, the largest epidemiologic cohort studied to date with over 1 million patients [10]. This included the mean patient age (13 $[ \pm 2]$ years old in this study versus $13[ \pm 3]$ years old in Kessler et al.'s study), gender distribution (proportion of male patients, $75 \%$ versus $79 \%$ ), lesion laterality (proportion of right knees, $42 \%$ versus 50\%), and lesion location (proportion of medial femoral condyle lesions, $62 \%$ versus $64 \%$ ). Other studies have also reported a male:female ratio of 2 to $3: 1$ [22], which is consistent with the ratio observed in our cohort, and a frequency of bilateral involvement between $3 \%$ and $33 \%$, again comparable to our observed frequency of $20 \%$ [7, 10, 16, 22]. Regarding the latter facet of survey studies, nonresponse bias may have contributed to a nonrepresentative patient cohort, particularly if those families with a history of knee problems were more likely to respond. Although this may have led to an overestimation of the percentage of patients with a positive family history, thus affecting the results for our first research question, it is less likely that such a response pattern would influence our findings with regard to our second research question, because the proportion of positive family histories would still be higher in one group if phenotypic potency was indeed related to familial inheritance. Finally, although there was no appreciated difference in gender distribution, included patients were approximately 1 year younger than survey nonresponders, which is of unclear clinical significance. We were also unable to assess phenotypic potency or race/ethnicity without a retrospective chart review of all nonresponders, which was not performed.

Several factors may have contributed to an overrepresentation of a positive family history in our cohort. Recall bias may have affected the proportion of patients reporting a positive family history for $\mathrm{OCD}$, particularly because knee injury as a youth is common, whereas OCD is a relatively rare disease that may have been misdiagnosed before the routine use of advanced imaging and arthroscopy. To minimize this potential effect, each reported positive history was further queried regarding clinical and treatment details to assess consistency with a typical OCD disease course. Any unclear cases and/or those with insufficient corroborating history (eg, "my father had unilateral knee surgery at 17, but I don't know why"; "my uncle had knee surgery between 18 and 25, but we're not sure if it was trauma or sportsrelated") were not counted. Additionally, to control for assessment bias, all surveys were administered and/or interpreted by the first author, who was blinded to clinical history at the time of survey administration and initial data entry and had adequate clinical experience managing OCD to appropriately differentiate it from other common traumatic and sports-related injuries of the knee. Given its preliminary nature, self-reported positive histories were not confirmed by direct contact with affected relatives, review of relevant medical records, or in-person clinical and/or radiographic screening. Finally, as an urban center, we likely had an increased proportion of patients of African American ethnicity with Kessler et al. suggesting that black patients had the highest odds ratio of OCD of the knee compared with all other ethnic groups [10].

Although our findings suggest that OCD has a similar inheritance pattern across all phenotypic severities of lesions, it is possible that our sample size may not have been large enough to adequately detect differences among the subsets. Other limitations in this study may have included our definition of phenotypic potency. In comparison to a more objective measure of radiographic disease severity or arthroscopic findings, we used bilateral disease and/or multiple procedures on the same lesion as indicators of high phenotypic potency. Thus, our 
definition may have been overly sensitive to aggressive treatment and/or increased screening of the contralateral limbs. Nevertheless, the frequency of bilateral disease in our study was within range of previous studies $(20 \%$ versus $3 \%$ to $33 \%[7,10,16,22]$ ), and the entire cohort was managed by a single surgeon with extensive clinical and operative expertise managing pediatric OCD (TJG). Per this surgeon's clinical practice, all patients with an intact lesion are first treated with a trial of conservative therapy. Although a large majority of our cohort was treated surgically (75 of 103 [73\%]), this may be indicative of the stronger relationship patients develop with their surgeon after being managed operatively and followed postoperatively, making these patients more likely to respond to a mailed survey and/or phone call. Furthermore, our findings were within the range of previous studies describing operative frequency in pediatric OCD of $(34 \%-74 \%[2,7,26])$. Although we did not have a "normal" population to compare our frequency of positive family history to, a number of previous studies have described the general incidence of OCD in the broader pediatric population, including Kessler et al.'s robust epidemiologic study of an integrated health system with more than 1 million patients [10]. Finally, because we did not collect the total number of immediate and extended family members for each patient, we were unable to determine a specific inheritance pattern and were only able to conclude that OCD appears to be a familial disease.

Fourteen percent (14 of 103) of treated patients in our series had an immediate or extended family member with a history of OCD, which seems greater than the general population incidence. Linden [13] identified $0.02 \%$ to $0.03 \%$ based on analysis of knee radiographs, whereas Kessler et al. [10] reported a $0.01 \%$ incidence in their recent epidemiologic survey. Conversely, Bradley and Dandy [3] reported an incidence of $1.2 \%$ based on an analysis of OCD lesions seen on the femoral condyles during 5000 knee arthroscopies, and Marsden and Wiernik found an incidence of $4 \%$ in all knee radiographs reviewed from 18,405 radiographs of male patients in a military hospital. To our knowledge, no previous studies have formally assessed the incidence of OCD in relatives of patients who had previously been treated for OCD of the knee, although some reports have described case series of lesions among twins [5, 9, 15, 17, 19, 21] and families with genetic syndromes [1, 24]. In each of these reports, including ours, the incidence among family members was higher than expected based on the incidence of OCD in the general population, suggesting an underlying genetic component to disease development. Although our incidence may be a mild overestimation, as discussed previously, it is nevertheless much higher than these previous population reports, providing further evidence in support of ongoing genetic investigation of OCD. However, although our data do support the genetic etiology hypothesis for OCD lesions, it does not help to specifically identify a potential genetic link. Thus, more thorough genetic analysis is warranted to further this investigation. Some of this work is already ongoing; the senior author recently completed a genome-wide association study (GWAS) to uncover candidate loci associated with the pathogenesis of OCD [27]. By comparing blood samples from 209 individuals with OCD to $1855 \mathrm{popu}-$ lation-matched control subjects, 35 single nucleotide polymorphisms (SNPs) were identified at several loci that suggested an association with OCD lesions. Further sequencing of a larger cohort is needed to determine if any of these identified SNPs are associated with OCD. Although a GWAS is capable of identifying genetic variants that are common in the population, it is possible that genetic variants associated with OCD are a set of private mutations in families. Thus, whole-exome sequencing of multicase families should also be investigated to uncover potential private mutations contributing to OCD. Therefore, this study supports ongoing efforts at whole-exome sequencing and/or other gene-level techniques to determine private mutations resulting in heritable OCD lesions.

With the numbers available, we also did not identify a higher proportion of family members with a positive history of OCD in those patients with phenotypically potent lesions. To our knowledge, no previous studies looked at potency of OCD lesions with regard to the presence or absence of a positive family history of disease. This study included two sets of siblings each counted as separate patients, including one set of monozygotic twins previously described in a report from our institution (Ganz et al. [5]; Case 2, with Twin B developing contralateral disease during followup of his initial unilateral lesion that was subsequently treated with two surgical procedures). Interestingly, despite an identical genetic substrate and similar activity levels, phenotypic potency was not the same for these monozygotic twins. Nevertheless, because this work is preliminary in nature and represents only one set of twins, future investigation should continue to investigate the precise relationship between family history and OCD phenotypic penetrance with a specific emphasis on genome sequencing and increasing the number and demographic diversity of patients in each of the phenotypic categories.

In conclusion, our data provide preliminary support for a familial inheritance pattern for OCD, suggesting that further clinical, biologic, and genomic investigation might help to improve our understanding of who is at highest risk 
for OCD and what moderating genetic and other factors might influence their disease severity and risk of progression. Furthermore, our data suggest that expanded patient education and screening regarding inheritance patterns might enhance identification of potential familial disease burden and improve access to timely and appropriate treatment.

\section{References}

1. Al Kaissi A, Klaushofer K, Grill F. Osteochondritis dissecans and osgood schlatter disease in a family with stickler syndrome. Pediatr Rheumatol Online J. 2009;7:4.

2. Backes JR, Durbin TC, Bentley JC, Klingele KE. Multifocal juvenile osteochondritis dissecans of the knee: a case series. $J$ Pediatr Orthop. 2014;4:453-458.

3. Bradley J, Dandy DJ. Osteochondritis dissecans and other lesions of the femoral condyles. J Bone Joint Surg Br. 1989;3:518-522.

4. Gans I, Grant S, Ganley T. The genetic nature of osteochondritis dissecans: a systematic review and call for improved studies. University of Pennsylvania Orthopaedics Journal. 2013:14-16.

5. Gans I, Sarkissian EJ, Grant SF, Ganley TJ. Identical osteochondritis dissecans lesions of the knee in sets of monozygotic twins. Orthopedics. 2013;12:e1559-1562.

6. Green JP. Osteochondritis dissecans of the knee. J Bone Joint Surg Br. 1966;1:82-91.

7. Hefti F, Beguiristain J, Krauspe R, Moller-Madsen B, Riccio V, Tschauner C, Wetzel R, Zeller R. Osteochondritis dissecans: a multicenter study of the European Pediatric Orthopedic Society. $J$ Pediatr Orthop B. 1999;4:231-245.

8. Jackson GC, Marcus-Soekarman D, Stolte-Dijkstra I, Verrips A, Taylor JA, Briggs MD. Type IX collagen gene mutations can result in multiple epiphyseal dysplasia that is associated with osteochondritis dissecans and a mild myopathy. Am J Med Genet A. 2010;4:863-869.

9. Jeong JH, Mascarenhas R, Yoon HS. Bilateral osteochondritis dissecans of the femoral condyles in both knees: a report of two sibling cases. Knee Surg Relat Res. 2013;2:88-92.

10. Kessler JI, Nikizad H, Shea KG, Jacobs JC Jr, Bebchuk JD, Weiss JM. The demographics and epidemiology of osteochondritis dissecans of the knee in children and adolescents. Am J Sports Med. 2014;2:320-326.

11. Konig F. The Classic: On loose bodies in the joint. 1887. Clin Orthop Relat Res. 2013;471:1107-1115.
12. Lee MC, Kelly DM, Sucato DJ, Herring JA. Familial bilateral osteochondritis dissecans of the femoral head. A case series. $J$ Bone Joint Surg Am. 2009;11:2700-2707.

13. Linden B. The incidence of osteochondritis dissecans in the condyles of the femur. Acta Orthop Scand. 1976;6:664-667.

14. Linden B, Telhag H. Osteochondritis dissecans. A histologic and autoradiographic study in man. Acta Orthop Scand. 1977;6:682-686.

15. Mackie T, Wilkins RM. Case report: osteochondritis dissecans in twins: treatment with fresh osteochondral grafts. Clin Orthop Relat Res. 2010;468:893-897.

16. Marsden C, Wiernik G. The incidence of osteochondritis dissecans. J R Army Med Corps. 1956;2:124-130.

17. Mei-Dan O, Mann G, Steinbacher G, Cugat RB, Alvarez PD. Bilateral osteochondritis dissecans of the knees in monozygotic twins: the genetic factor and review of the etiology. Am J Orthop (Belle Mead NJ). 2009;9:E152-155.

18. Mubarak S, Carroll N. Familial osteochondritis disecans of the knee. Clin Orthop Relat Res. 1979;140:131-136.

19. Onoda S, Sugita T, Aizawa T, Ohnuma M, Takahashi A. Osteochondritis dissecans of the knee in identical twins: a report of two cases. J Orthop Surg (Hong Kong). 2012;1:108-110.

20. Petrie PW. Aetiology of osteochondritis dissecans. failure to establish a familial background. J Bone Joint Surg Br. 1977;3: 366-367.

21. Richie LB, Sytsma MJ. Matching osteochondritis dissecans lesions in identical twin brothers. Orthopedics. 2013;9:e12131216.

22. Schenck RC Jr, Goodnight JM. Osteochondritis dissecans. J Bone Joint Surg Am. 1996;3:439-456.

23. Shea KG, Jacobs JC Jr, Carey JL, Anderson AF, Oxford JT. Osteochondritis dissecans knee histology studies have variable findings and theories of etiology. Clin Orthop Relat Res. 2013; 471:1127-1136.

24. Stattin EL, Tegner Y, Domellof M, Dahl N. Familial osteochondritis dissecans associated with early osteoarthritis and disproportionate short stature. Osteoarthritis Cartilage. 2008;8: 890-896.

25. Stougaard J. Familial occurance of osteochondritis dissecans. $J$ Bone J Surg, Br. 1964:542-543.

26. Weiss JM, Nikizad H, Shea KG, Gyurdzhyan S, Jacobs JC, Cannamela PC, Kessler JI. The incidence of surgery in osteochondritis dissecans in children and adolescents. Orthop J Sports Med. 2016;3:2325967116635515.

27. Yellin JL, Trocle A, Grant SF, Hakonarson H, Shea KG, Ganley TJ. Candidate loci are revealed by an initial genome-wide association study of juvenile osteochondritis dissecans. $J$ Pediatr Orthop. 2015 Sep 28. [Epub ahead of print] 